\title{
A Study on the Effects of the Ice Microphysics on Tropical Cyclones
}

\author{
Masanori Yamasaki \\ Research Institute for Global Change, Japan Agency for Marine-Earth Science and Technology, 3173-25 Showa-machi, \\ Kanazawa-ku, Yokohama 236-0001, Japan \\ Correspondence should be addressed to Masanori Yamasaki; mn-yama@lucky.odn.ne.jp
}

Received 18 December 2012; Revised 25 March 2013; Accepted 3 April 2013

Academic Editor: Richard Leaitch

Copyright (C) 2013 Masanori Yamasaki. This is an open access article distributed under the Creative Commons Attribution License, which permits unrestricted use, distribution, and reproduction in any medium, provided the original work is properly cited.

The effects of the ice microphysical processes on the development of weak vortices and tropical cyclones (TCs) are examined by numerical experiments with a nonhydrostatic model. Since it has been understood that the ice phase generally enhances the eyewall circulation in strong TCs because of additional heat release and insignificant effect of rainwater evaporation, this study focuses on the development of relatively weak vortices and TCs. Some past studies showed that the development is slower by the effects of the ice phase through cooling due to the melting of snow and graupel, whereas this study indicates that cooling due to evaporation of rainwater in the subcloud layer plays a much more important role in the slower development, and much more solid substances in the mid-troposphere, which are produced through the ice phase processes, contribute to more rainwater evaporation in the subcloud layer. The relative importance of many processes of the ice microphysics is also examined as a basis for future improvements of parameterization of the microphysical processes.

\section{Introduction}

This paper describes results from numerical experiments which have been made to get a better understanding of the effect (role) of ice microphysical processes in tropical cyclones (TCs) with the use of a nonhydrostatic model.

The essential mechanism of the formation (genesis), development, and the structure of TCs can be explained even without the consideration of the ice microphysical processes (hereafter, ice phase). In the 1960s, the development of TCs was explained by the concept of CISK (conditional instability of the second kind) of the type in which frictional convergence plays an essential role (Ooyama [1,2]; Charney and Eliassen [3]; Yamasaki [4, 5]; Rosenthal [6]; and others). In these studies, any cloud microphysical process (even warm rain process) was not taken into consideration. This CISK, which can be referred to as frictional convergence CISK, explains the eyewall circulation of TCs, as later recognized (Yamasaki [7-9]). The cloud microphysics is not important to this CISK.

The present author (Yamasaki $[7,10,11]$ ) identified another type of CISK in which frictionally controlled flow (other than frictional convergence) plays an important role. In this type of CISK (frictional flow CISK), cooling due to rainwater evaporation in the subcloud layer plays an important role through the successive formation of convective clouds, which is due to interaction of the outflow of cold pool air with the vortex-scale inflow of warm, moist air. The author has, in these 30 years, considered that even a numerical model only with the warm rain process can explain many essential aspects of TCs.

The effect of the ice phase on TCs was studied by Willoughby et al. [12] as an extension of Yamasaki [7, 10, 11, 13] which did not take account of the ice phase. According to Figures 3 and 7 in their paper, the development of a vortex (TC) is slower in the presence of the ice phase. This feature was mentioned by Lord et al. [14]. The same result was obtained by Sawada and Iwasaki [15]. The slower development was attributed to the cooling associated with the melting of solid particles.

It is important to remark that the ice phase effects are different, depending on the type of convective clouds in the vortex (TC). The effect in eyewall clouds is different from that in rainbands outside the eyewall. In the warm rain case, 
the author recognized clear difference between the effects of rainwater evaporation in the eyewall and outer convective clouds in TCs (Yamasaki [7]). In numerical experiments of Hurricane Bonnie (1998) by Zhu and Zhang [16], the development was slower in the warm rain case than in the cold rain case. This is because they treated a strong hurricane with an eyewall in which the maximum wind speeds were more than $30 \mathrm{~m} \mathrm{~s}^{-1}$.

In this study, we treat a vortex (TC) with a weaker intensity at the initial time of the numerical experiments. This is because the author has probably understood why the ice phase enhances the eyewall convection (and TC); it is enhanced through additional heat release compared with the warm rain case. One of the objectives of this study is to understand why the development of a weaker vortex (TC) is slower in the presence of the ice phase, as shown by Willoughby et al. [12] and others. One of the most important results is that precipitating (solid) particles are much more produced in the mid-troposphere in the cold rain case than precipitating (rainwater) particles in the warm rain case, and more cooling occurs in the subcloud layer, owing to more evaporation of precipitating rainwater. It is emphasized that more precipitating particles in the mid-troposphere and more cooling in the subcloud layer are responsible for slower development. The cooling in the melting layer is not the major cause. This recognition is different from that in the past studies.

In this study, a one-moment bulk model (without treatment of number concentrations of particles) is used for the cloud microphysics, although the author is also interested in other researchers' results from two-moment bulk models and bin models. The parameterization scheme used is described in Yamasaki [17], which is an improved version (Sato and Yamasaki [18-20]) of Lin et al. [21]. Some of the modifications from [21] are described in Section 2. Although it can be expected that the result concerning the slower development in the presence of the ice phase is not essentially modified by parameterization schemes, a better scheme will be desirable for general discussions of the ice phase effects in the future. As a step toward a better scheme, the relative importance of many cloud microphysical processes (about 40) involved in the one-moment bulk model is shown in this paper.

\section{Model and Experimental Design}

In this study, a one-moment bulk model is used, as mentioned in Section 1. It is based on Lin et al. [21], but several modifications are made. Three points among them are described in the following: (1) amount of cloud ice was diagnostically determined by Lin et al. [21]. Murakami [22] determined it prognostically (with production rate of cloud ice). However, cloud ice formation (depositional/sorptional nucleation) is restricted to ascending areas in his model. Sato and Yamasaki $[18,19]$, which are referred to as SY, proposed an assumption that cloud ice formation due to this process does not depend on the vertical motion directly. When the assumption of Lin et al. [21] was used, amount of cloud ice was too small (SY). This was improved by SY's assumption. (2) Lin et al. [21]
TABLE 1: The heights of the levels where the vertical velocity is predicted for a 30-layer model, the environmental temperature $T_{B}$, and the initial relative humidity $R_{H 0}$ at the center of the moist area $(x=0, y=-100 \mathrm{~km})$ are also shown.

\begin{tabular}{|c|c|c|}
\hline$z(\mathrm{~km})$ & $T_{B}(\mathrm{~K})$ & $R_{H 0}(\%)$ \\
\hline 24.0 & 199.2 & 30 \\
\hline 21.4 & 199.2 & 30 \\
\hline 19.1 & 199.2 & 33 \\
\hline 17.2 & 199.2 & 36 \\
\hline 15.6 & 200.5 & 40 \\
\hline 14.2 & 205.5 & 44 \\
\hline 13.0 & 213.0 & 48 \\
\hline 12.0 & 221.2 & 52 \\
\hline 11.1 & 229.0 & 56 \\
\hline 10.3 & 235.4 & 60 \\
\hline 9.6 & 240.9 & 63 \\
\hline 9.0 & 245.3 & 66 \\
\hline 8.5 & 249.1 & 69 \\
\hline 8.0 & 252.5 & 72 \\
\hline 7.5 & 256.1 & 75 \\
\hline 7.0 & 259.4 & 78 \\
\hline 6.5 & 262.4 & 80 \\
\hline 6.0 & 265.5 & 82 \\
\hline 5.5 & 268.4 & 83 \\
\hline 5.0 & 271.3 & 84 \\
\hline 4.5 & 274.3 & 85 \\
\hline 4.0 & 277.2 & 86 \\
\hline 3.5 & 280.1 & 87 \\
\hline 3.0 & 282.7 & 88 \\
\hline 2.5 & 285.5 & 89 \\
\hline 2.0 & 288.2 & 90 \\
\hline 1.5 & 290.9 & 92 \\
\hline 1.0 & 293.6 & 92 \\
\hline 0.6 & 296.0 & 90 \\
\hline 0.3 & 298.0 & 88 \\
\hline 0.0 & 300.0 & 85 \\
\hline
\end{tabular}

TABLE 2: Latitudinal distribution of the sea surface temperature used in the numerical experiments.

\begin{tabular}{lc}
\hline Latitude & SST \\
\hline $35 \mathrm{~N}$ & 296.0 \\
$30 \mathrm{~N}$ & 299.0 \\
$25 \mathrm{~N}$ & 301.0 \\
$20 \mathrm{~N}$ & 302.0 \\
$10 \mathrm{~N}$ & 302.0 \\
$5 \mathrm{~N}$ & 301.0 \\
$\mathrm{EQ}$ & 300.0 \\
$5 \mathrm{~S}$ & 298.0 \\
\hline
\end{tabular}

used snow and hail (in addition to cloud ice) as three classes in the ice phase. SY used snow (snow aggregate rather than snowflake) and graupel because graupel exists in TCs. (3) Autoconversion from snow to hail and accretion of snow by 
rainwater $(0,15 \mathrm{~km})$, central surface pressure, and maximum wind speed
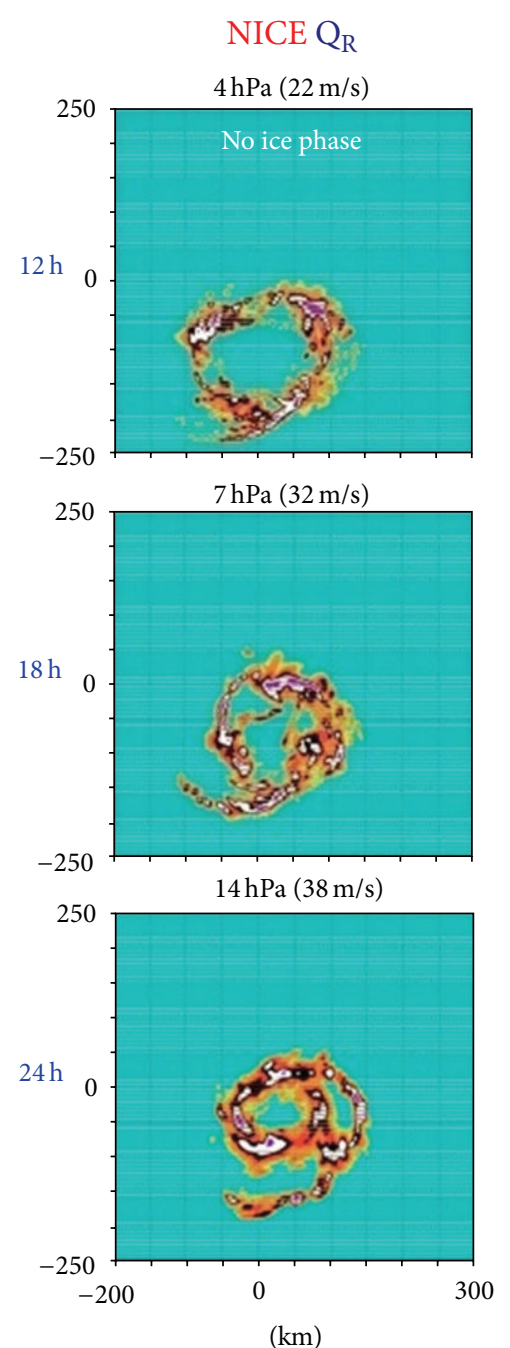

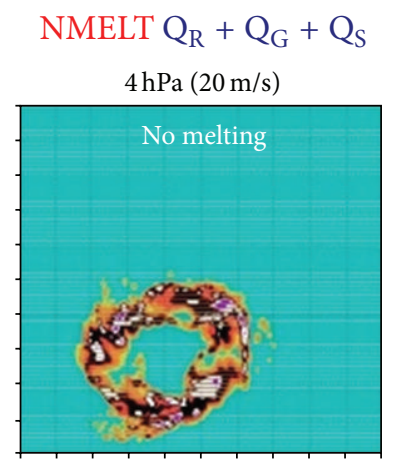

$6 \mathrm{hPa}(25 \mathrm{~m} / \mathrm{s})$

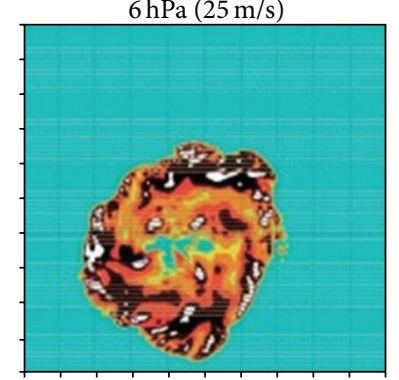

$10 \mathrm{hPa}(24 \mathrm{~m} / \mathrm{s})$

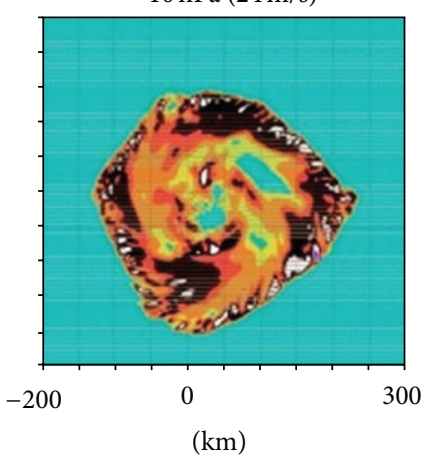

ICE $Q_{R}$

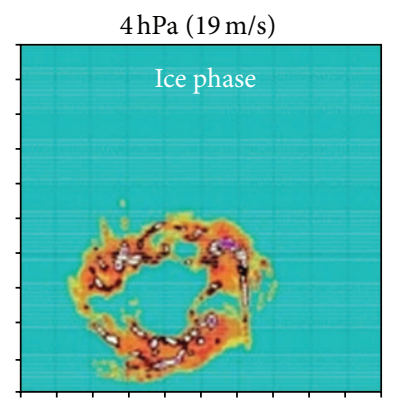

$6 \mathrm{hPa}(21 \mathrm{~m} / \mathrm{s})$

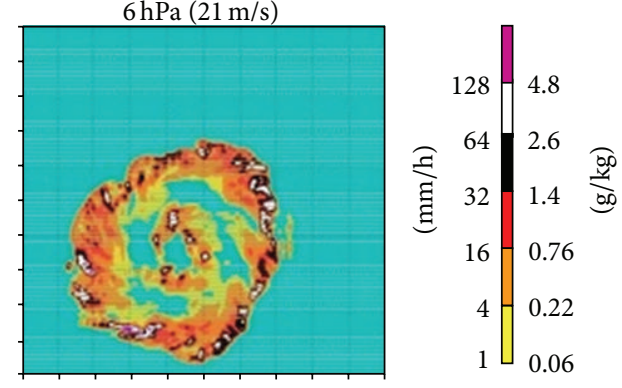

$10 \mathrm{hPa}(24 \mathrm{~m} / \mathrm{s})$

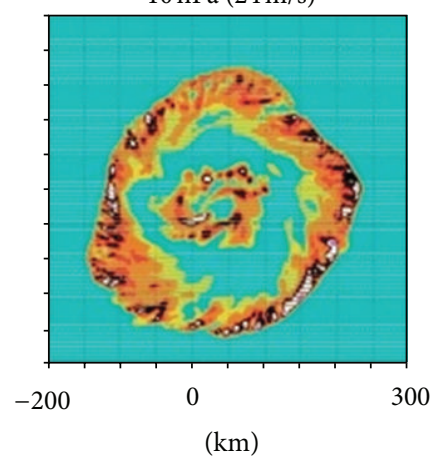

FIGURE 1: Comparison of the mixing ratio of rainwater at $0.15 \mathrm{~km}$ height, the central surface pressure and the maximum wind speed at $1.25 \mathrm{~km}$ in case NICE, case NMELT, and case ICE at $12 \mathrm{~h}, 18 \mathrm{~h}$, and $24 \mathrm{~h}$. The surface pressure is given by the negative value of its anomaly from the environment.

hail were taken into account by Lin et al. [21], whereas it was assumed in SY that these two processes (conversions from snow to graupel in this study) should not occur. Under this condition, the main process of graupel formation is accretion of cloud water by snow (riming). The difference between the distributions of snow and graupel in the two models (with and without autoconversion and accretion) was studied [20] with an axisymmetric model. This problem remains to be studied with a three-dimensional model in the future. For further details as to modifications (1) (3) and other microphysics, readers are referred to Yamasaki [17].

Dynamical and thermodynamical aspects of the model used are described in [17]. It is desirable to use a cumulusconvection-resolving model with a horizontal grid size of $1 \mathrm{~km}$ or less. However, a $2 \mathrm{~km}$ grid is used in this study because the essence of the problems treated in this study can be discussed by this grid size. This grid size was used by Sawada and Iwasaki [15]. In this study, this grid size is used for a $600 \mathrm{~km}$ square domain. Outside this domain, a nonuniform, coarse grid is used. The whole domain covers the $4,000 \mathrm{~km}$ square. As for the vertical, a 30-layer model is used. The levels where the vertical velocity is predicted are shown in Table 1.

The environmental flow and other disturbances such as a subtropical high are not included because they are not essential to the understanding of ice phase effects. The basic-state (environmental) temperature $T_{B}$, which nearly corresponds to that of the tropical atmosphere in north western Pacific in summer, and relative humidity $R_{H 0}$ at the moist center $(x=0, y=-100 \mathrm{~km})$ are shown in Table 1. (All physical quantities except the vertical velocity are predicted at the midlevels between the two levels shown in Table 1, as usually done in nonhydrostatic models.) The relative humidity is taken to be $80 \%$ of these values in areas of $x>800 \mathrm{~km}, x<-800 \mathrm{~km}, y>200 \mathrm{~km}$, and $y<-900 \mathrm{~km}$. 
rainwater $(0,15 \mathrm{~km})$, central surface pressure, and maximum wind speed
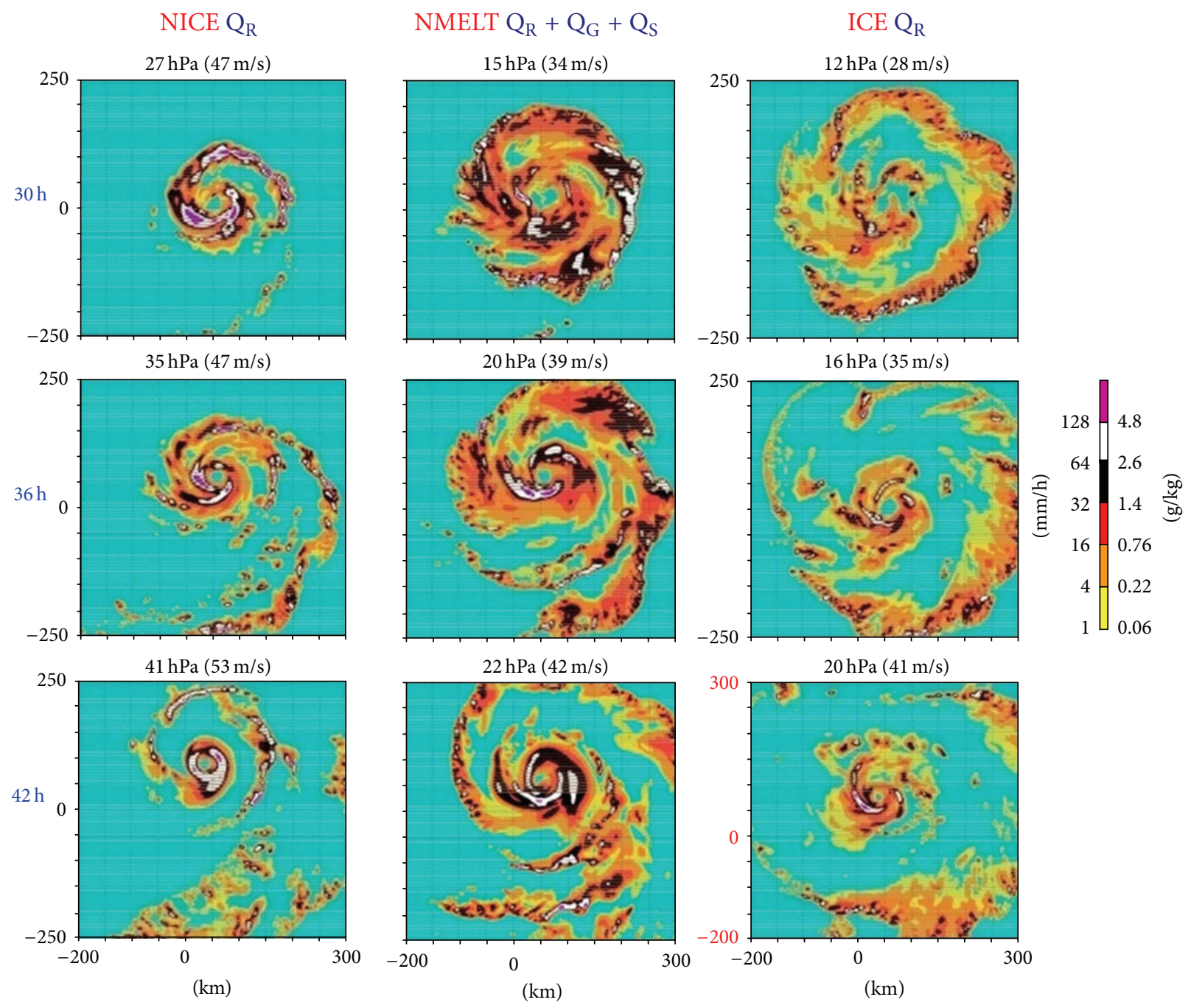

Figure 2: Same as Figure 1 but for $30 \mathrm{~h}, 36 \mathrm{~h}$, and $42 \mathrm{~h}$.

The distribution in the $x$ - and $y$-directions is taken to be the square of cosine in the square area indicated by the above four values. The origin $y=0$ is taken to be $15 \mathrm{~N}$. The sea surface temperature SST used in this study is shown in Table 2. The SSTs between the two latitudes are linearly interpolated.

An axisymmetric vortex is given at the initial time, as was done in the previous studies, although results from other initial conditions will be reported in a separate paper. The latitudinal variation of the Coriolis parameter is taken into account in this study because this contributes to the vortex development through low-level inflow enhanced by formation of asymmetry (such as spiral rainband). The maximum wind speed is taken to be $10 \mathrm{~m} \mathrm{~s}^{-1}$, and it is located at the $200 \mathrm{~km}$ radius. Under this initial condition, convection is initiated by frictional convergence, and it takes a ring shape. It propagates outward because the maximum wind speed of $10 \mathrm{~m} \mathrm{~s}^{-1}$ is given. Once convection becomes active, frictional convergence is not important (until eyewall formation stage), and the cooling due to rainwater evaporation in the subcloud layer plays an important role. Frictional flow CISK, as mentioned in Section 1, works at the stage before eyewall formation. This was recognized in the previous studies (Yamasaki $[7,10,11]$ ).

In order to understand the effects of the ice phase, three numerical experiments have been performed. In the first case (case NICE), ice phase is not included (warm rain case). In the second case (case NMLT), the ice phase is included, but it is assumed that the melting of snow and graupel does not occur; snow and graupel fall to the sea surface while these can sublimate. That is, rainwater is not produced through the melting of snow and graupel, although it can exist owing to other processes. This case is different from that of Sawada and Iwasaki [15] in which only the cooling rate due to melting was assumed to be 0 . In the third case (case ICE), all ice phase processes are taken into account. 

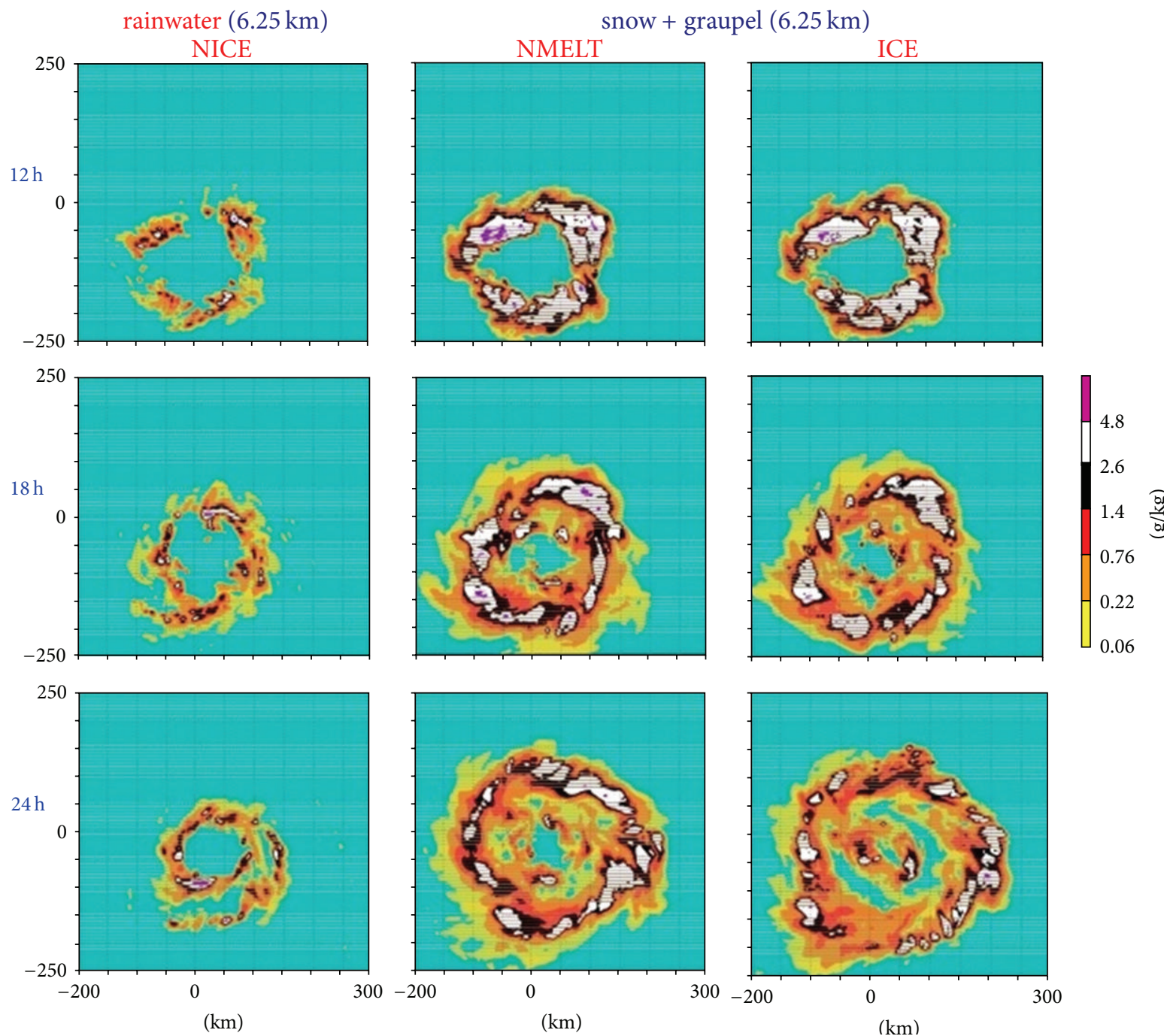

FIGURE 3: Comparison of the mixing ratio of precipitating substance; rainwater in case NICE, and the sum of snow and graupel at $6.25 \mathrm{~km}$ height at $12 \mathrm{~h}, 18 \mathrm{~h}$, and $24 \mathrm{~h}$.

\section{Effects of the Ice Phase}

In this section, results from the three numerical experiments are compared to understand the effects of the ice phase. Figure 1 shows the mixing ratio of rainwater (or total precipitating substance) at a height of $150 \mathrm{~m}$ (or precipitation intensity at the surface) at $12 \mathrm{~h}, 18 \mathrm{~h}$, and $24 \mathrm{~h}$ in the three cases mentioned in Section 2. The central surface pressure and the maximum wind speed at a $1.25 \mathrm{~km}$ height are indicated by numerals. The surface pressure is given by the negative value of its anomaly from the environment. Figure 2 shows the results at $30 \mathrm{~h}, 36 \mathrm{~h}$, and $42 \mathrm{~h}$. (The area of the right-bottom panel is different from that in other panels.)

Comparison of cases NICE and ICE confirms that the vortex (TC) development is slower when the ice phase is included, as pointed out by the previous studies. Comparison of NMELT and ICE shows that the vortex development is slightly slower in ICE. The difference is very small and even insignificant. That is, the effect of the melting of graupel and snow on the vortex development is very small (in terms of the central surface pressure and the maximum wind speed). On the contrary, the distribution of precipitation substance is very affected by the melting. The precipitation amount is very much larger in NMELT than in ICE. Quantitative discussion will be made later (Table 3).

As mentioned in Section 2, convection is initiated by frictional convergence. It takes a ring shape and propagates outward at the early stage. One of the important differences between case NICE and the other two ice cases (NMELT and ICE) is that the speed of the outward propagation of the convective ring is much larger in the two ice cases. The faster propagation makes the low-level pressure gradient smaller, and the wind intensification becomes slower. In contrast, in case NICE, the convective ring is destroyed, and it takes a spiral-shape at the earlier stage. (The reason why asymmetry becomes notable earlier in case NICE remains to be understood.) As a result, the low-level air with high equivalent potential temperature tends to enter easily the central area of 

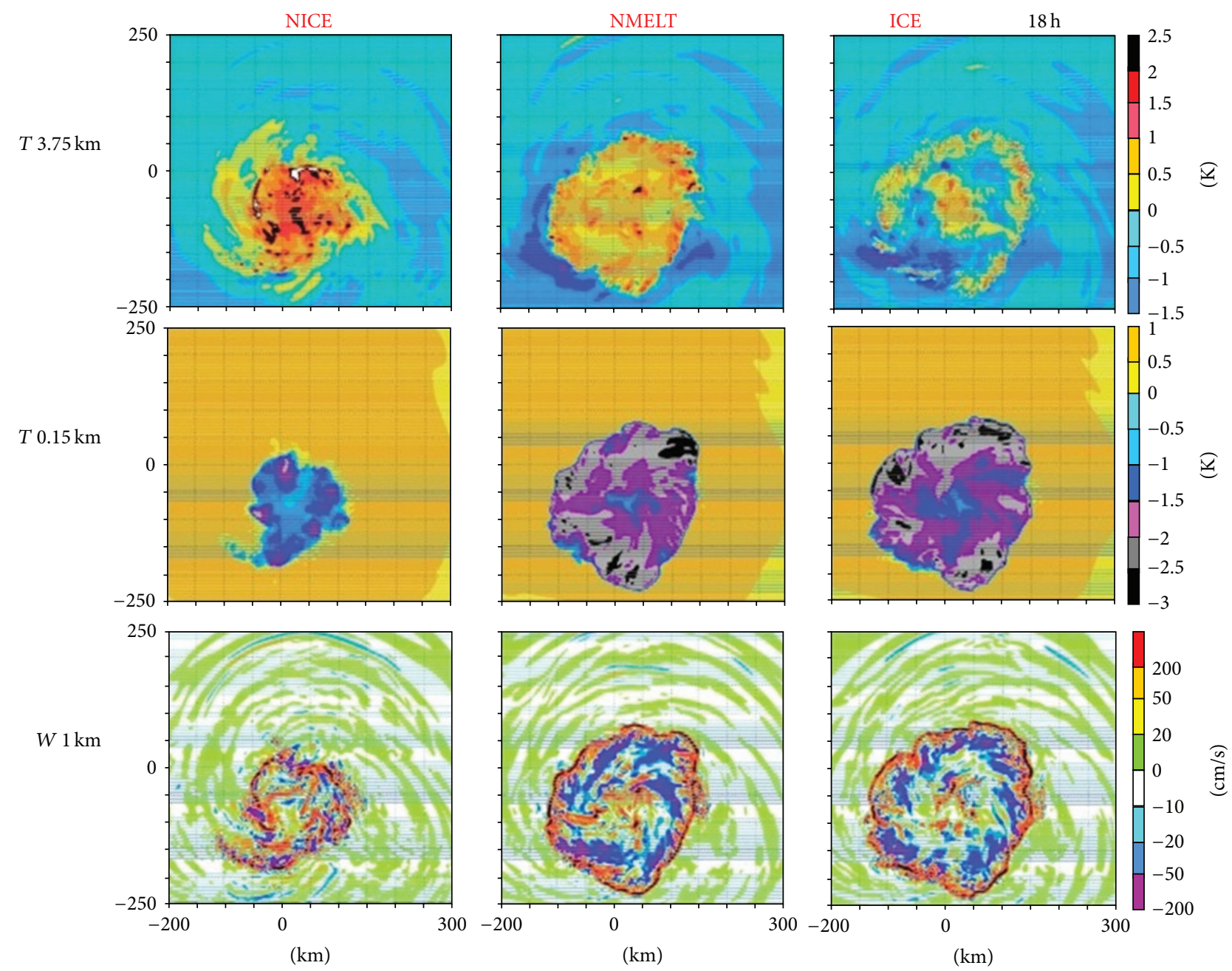

FIGURE 4: Comparison of the temperature anomaly at $3.75 \mathrm{~km}$ and $0.15 \mathrm{~km}$ and the vertical velocity at $1.0 \mathrm{~km}$ in case NICE, case NMELT, and case ICE at $18 \mathrm{~h}$.

the vortex, and convection in an area near the vortex center is more active. For these reasons, the development of the vortex in NICE is faster than in the two ice cases. In case NMELT, the outward-propagating convection becomes spiral rainbands at the later stage. On the other hand, in case ICE, it maintains nearly the ring shape for a somewhat long period, becomes asymmetric in some time, and decays eventually. New convection in an area near the vortex center becomes an eyewall cloud. The behaviors of the eyewall and spiral rainbands after $42 \mathrm{~h}$ will be shown later (Figure 5).

It is important to examine the amount of the precipitation substance in the mid-troposphere to understand why the vortex development is slower in the two ice cases. Figure 3 shows the mixing ratio of rainwater in case NICE and the total of snow and graupel in cases NMELT and ICE at $6.25 \mathrm{~km}$ at three selected times $12 \mathrm{~h}, 18 \mathrm{~h}$, and $24 \mathrm{~h}$. It is noteworthy that the precipitation amount in NMELT and ICE is much larger than in NICE. Larger amount of evaporation and sublimation in their falling process causes a stronger cold pool in the subcloud layer, as shown later (Figure 4). This contributes to faster outward propagation of the convective ring. It can also be seen from this figure that the melting of graupel and snow does not essentially alter the amount of precipitation substance in the mid-troposphere. That is, the melting does not play an active role in convective activity at this stage.

Figure 4 shows temperature deviations at $3.75 \mathrm{~km}$ (upper panels) and $0.15 \mathrm{~km}$ (middle panels) and vertical velocity at $1.0 \mathrm{~km}$ (bottom panels) in the three cases. It can be seen that the low-level downdraft (indicated by blue and purple colors in the bottom panels) and negative temperature deviation near the surface (strong cold pool indicated by grey and black colors in the middle panels) in the two ice cases are much larger than those in case NICE. That is, the ice phase produces much stronger downdraft and cold pool in the subcloud layer, which contributes to the faster speed of the outward propagation of the convective ring. Comparison of ICE with NMELT indicates that the melting produces significantly lower temperatures in the melting layer, as expected, whereas its effects on the intensities of the downdraft and cold pool in the subcloud layer are small. Therefore, the central surface pressure and the maximum wind speed are not much affected by the melting. 


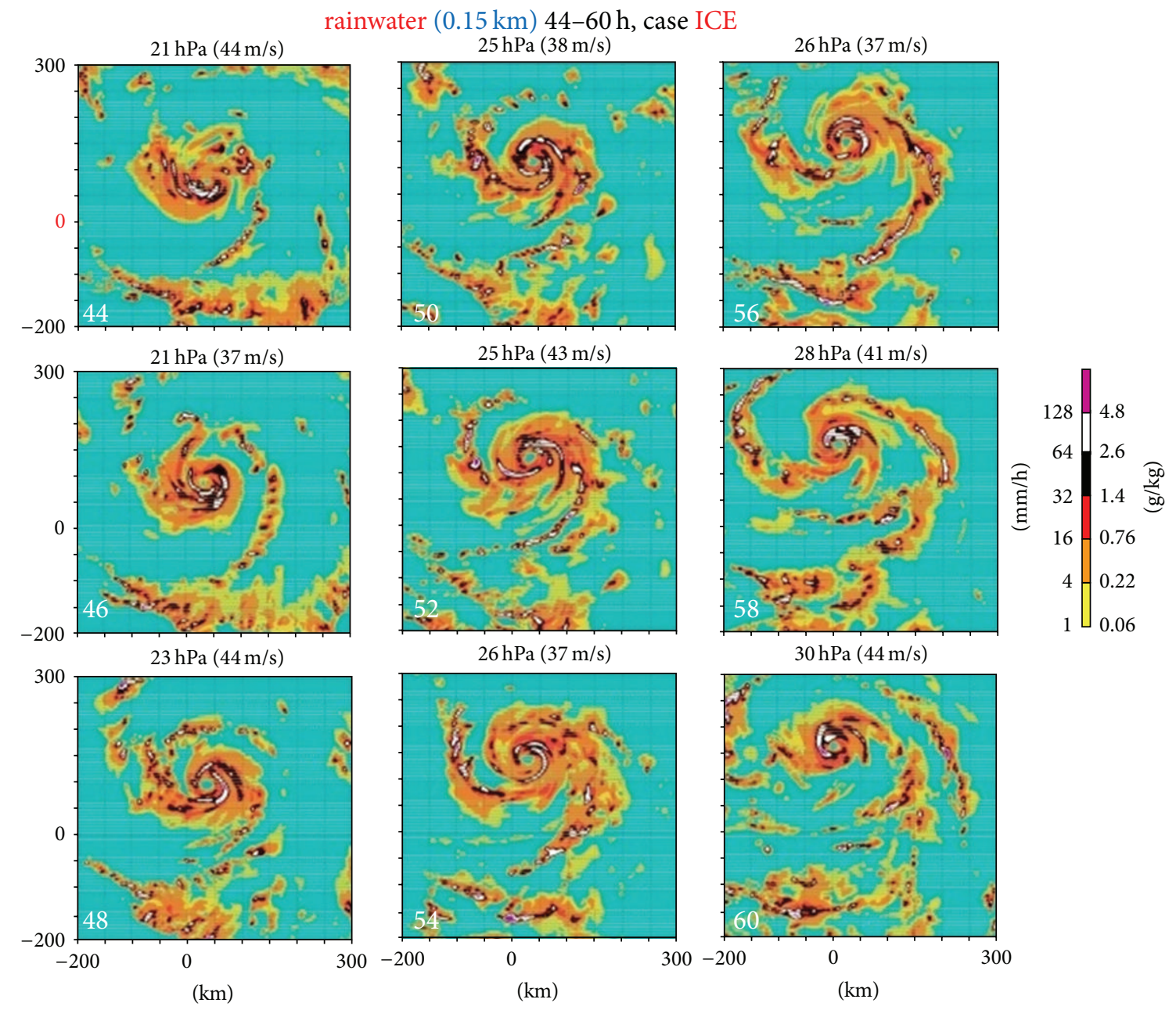

FIGURE 5: Rainwater mixing ratio at $0.15 \mathrm{~km}$, the central surface pressure, and the maximum wind speed at $1.25 \mathrm{~km}$, at a time interval of 2 hours from $44 \mathrm{~h}$ to $60 \mathrm{~h}$ in case ICE.

TABLE 3: Amount of rainwater or the sum of snow and graupel at $6.25 \mathrm{~km}$ and $0.15 \mathrm{~km}$ and Cooling due to evaporation (sublimation) in the lower layer below $3 \mathrm{~km}$ height. The numerals are given in certain unit.

\begin{tabular}{lccc}
\hline & \multicolumn{2}{c}{$Q_{R}$ or $Q_{S}+Q_{G}$} & $\begin{array}{c}\text { Cooling due to evaporation } \\
\text { (sublimation) }\end{array}$ \\
\hline NICE & $6.25 \mathrm{~km}$ & $0.15 \mathrm{~km}$ & 43 \\
NMELT & 12 & 15 & 73 \\
ICE & 73 & 23 & 63 \\
\hline
\end{tabular}

In order to further understand the effects of the ice phase and the melting, amount of rainwater or the sum of snow and graupel at $6.25 \mathrm{~km}$ and $0.15 \mathrm{~km}$ and cooling due to evaporation and sublimation in a lower layer below a height of $3 \mathrm{~km}$ are shown in Table 3. The numerals are given by certain units. (Only the relative magnitude is important.) The most important result from this table is that the amount of the precipitation substance in the mid-troposphere is much larger in the two ice cases than in case NICE, as also seen in
Figure 3. Nevertheless, amount of low-level rainwater in case ICE is almost the same as that in case NICE. This means that amounts of rainwater evaporation and evaporative cooling are significantly larger in ICE than those in NICE, as indicated by this table. This produces strong downdraft and cold pool in ICE (Figure 4), which contributes to faster propagation of the convective ring, as mentioned above. As for the melting effect, it is not seen in the mid-troposphere. The melting does not play an active role in convective activity, as mentioned. On the contrary, the melting contributes to more evaporation of rainwater and to less precipitation at the surface. The surface precipitation amount and pattern are significantly affected by the melting (Figures 1 and 2). Nevertheless, the melting effects on the intensity of the downdraft, cold pool, and vortex are small because the difference between the cooling amounts (due to evaporation and sublimation) in the lower layer in ICE and NMELT is small, as shown in this table. (The difference between the precipitation amount at the midtroposphere and that near the surface is larger in ICE than in NMELT, whereas cooling is less in ICE. This is because more sublimation cooling occurs in NMELT.) 

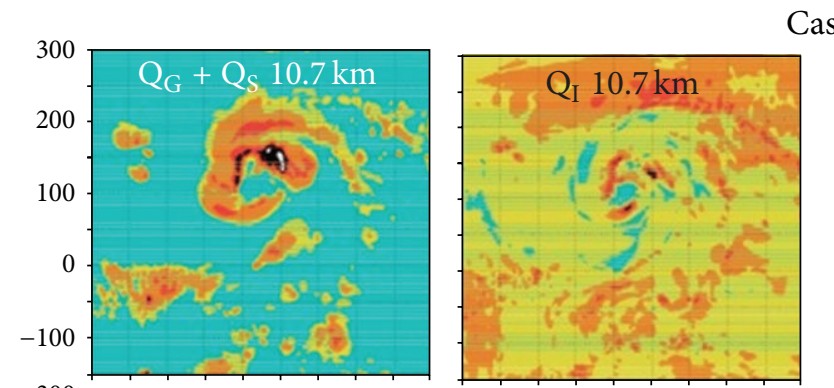

Case ICE

$58 \mathrm{~h}$
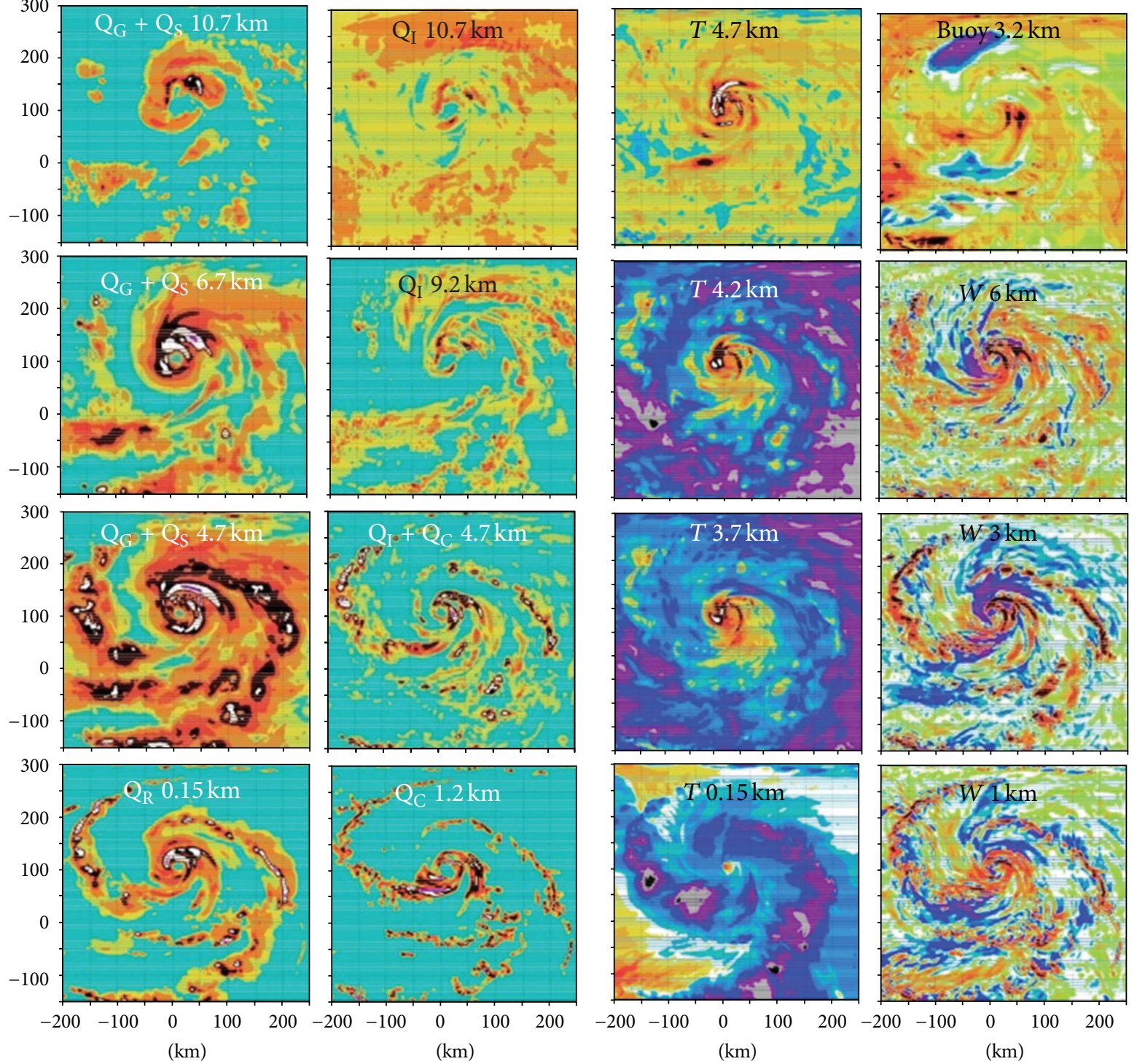

(a)
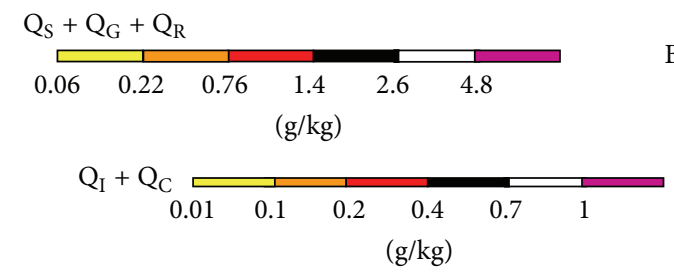

Buoy

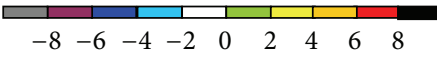

(K)

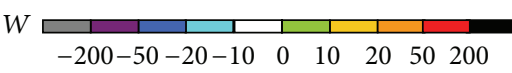

$(\mathrm{cm} / \mathrm{s})$

$T(3-5 \mathrm{~km})$

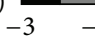

(K)

$T(0.15 \mathrm{~km})$

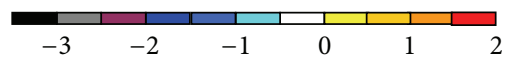

(K)

(b)

FIGURE 6: Mixing ratios of precipitating and nonprecipitating water substances, temperature anomaly, a measure of buoyancy of the rising air, and vertical velocity at various levels at $58 \mathrm{~h}$ in case ICE. 

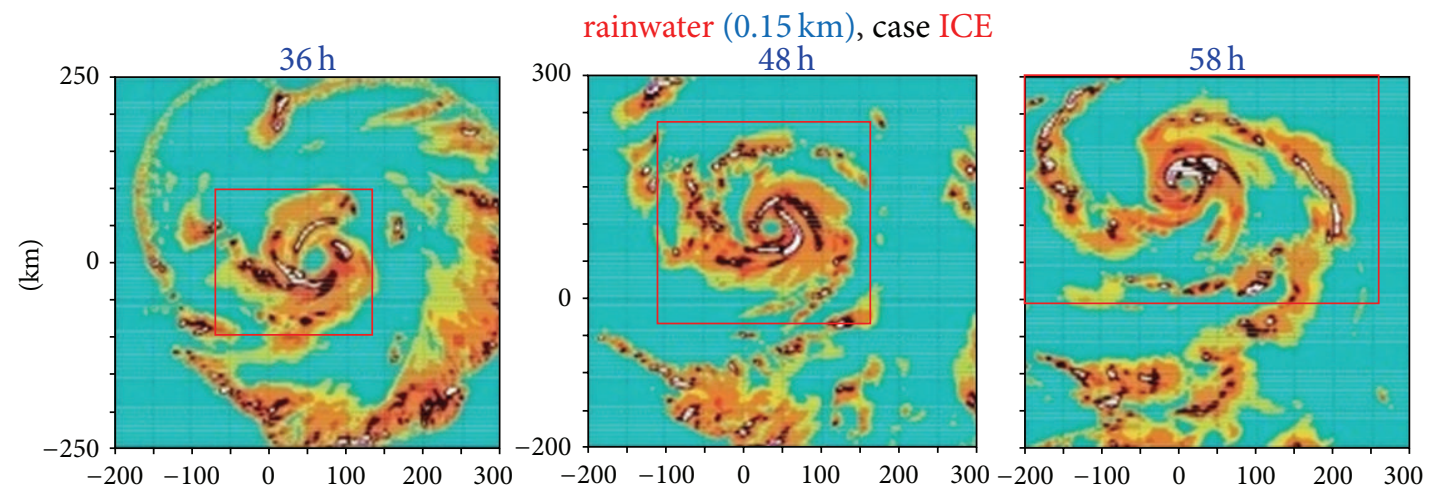

FIGURE 7: Rainwater mixing ratio at $0.15 \mathrm{~km}$ at $36 \mathrm{~h}, 48 \mathrm{~h}$, and $58 \mathrm{~h}$ in case ICE. The red square indicates the area where the magnitudes of cloud physical processes (Figures 9, 10, and 11) are averaged.

The behaviors of the eyewall and spiral rainbands in case ICE are shown at a time interval of 2 hours from $44 \mathrm{~h}$ to $60 \mathrm{~h}$ in Figure 5. Comparison of Figure 5 with Figures 1 and 2 indicates that the realization of the typical structure of TCs having the eyewall and spiral rainbands is delayed in ICE compared to NMELT, although significant differences are not found in the central surface pressure and the maximum wind speed (not shown for NMELT).

It can be seen from Figures 2 and 5 that the sizes of the eyewall and eye in NICE and ICE do not show any significant difference. This feature is also seen at the mature stage (not shown) and is different from that described by Sawada and Iwasaki [15], although further studies are needed to confirm this difference.

In order to see more detailed structure of the computed TC in case ICE and the degree of validity of the model used in this study, the mixing ratio of precipitating substance, that of nonprecipitating substance, and temperature anomaly at 4 selected levels; a measure of buoyancy which the air rising from the subcloud layer acquires at $3.2 \mathrm{~km}$ (defined as the difference between the equivalent potential temperature in the boundary layer and its saturation value at a height of $3.2 \mathrm{~km}$ ); and vertical velocity at 3 selected levels are shown in Figure 6. The selected time is 58 hours when the TC is still developing and spiral rainbands show distinct features. This figure shows that the amount of precipitating substance is largest just above the melting layer, as expected. The temperature anomaly at this level is positive, and that in the melting layer is negative except for the eye-eyewall area. The negative anomaly near the surface is distinct in the spiral band area. The vertical velocity field suggests the existence of internal gravity waves of small scales. Negative buoyancy of the rising air can be seen to the northwest of the eyewall and between spiral rainbands in the southwestern portion of the area shown. A very small eye can be identified in the field of nonprecipitating substance. Needless to say, this figure is merely one example of the structure of the computed TCs, and the structure has a wide variety. Although essential features of the TC structure are simulated, the degree of the model validity remains to be clarified for its improvement.

\section{Microphysical Processes}

In this section, we examine the relative importance of many microphysical processes involved in the one-moment bulk parameterization in case ICE. For this discussion, low-level rainwater distributions at $36 \mathrm{~h}, 48 \mathrm{~h}$, and $58 \mathrm{~h}$ are reproduced in Figure 7. Figure 8 shows the west-east vertical sections of the mixing ratio of cloud ice + cloud water, that of snow + graupel + rainwater, temperature deviation, radial velocity, tangential velocity, and vertical velocity at $48 \mathrm{~h}$. The vertical section crosses the eye (middle panel of Figure 7). Although these figures may show somewhat unrealistic aspects of these fields, the essential features of the structure of observed TCs are simulated. Needless to say, some details of the simulated structure (as well as that of observed TCs) change significantly with time.

Figure 9 shows the vertical cross-sections of the mixing ratio of snow (upper-left), and eight microphysical processes related to snow at $48 \mathrm{~h}$. (Colors of black, red, dark yellow, yellow, yellow green, green, and cyan for the microphysical processes indicate values of more than $50,20,10,5,2,1$, and 0.1 , resp., in some unit.) The left, central, and right numerals indicate values averaged over an area enclosed by the red square at $36 \mathrm{~h}, 48 \mathrm{~h}$, and $58 \mathrm{~h}$ in Figure 7, respectively. As is well known, snow is formed through autoconversion from cloud ice $\left(\mathrm{S}_{\mathrm{I}}(\right.$ auto $\left.)\right)$. Once some amount of snow is formed, increase of snow is primarily due to accretion of cloud ice by snow $\left(S_{I}\right)$, accretion of cloud water by snow $\left(S_{c}\right)$, and deposition of water vapor $\left(S_{V}(\right.$ dep $\left.)\right)$. The effects of accretion of snow $\left(S_{R}(R S)\right)$ and cloud ice $\left(S_{R}(R I)\right)$ by rainwater are small. Snow is converted to water vapor by sublimation $\left(\mathrm{V}_{\mathrm{S}}(\mathrm{sub})\right)$ and to rainwater by melting $\left(\mathrm{R}_{\mathrm{S}}(\mathrm{melt})\right)$. Other small contributing processes are not shown in the figure.

Figure 10 shows the vertical cross sections of the mixing ratio of graupel (upper-left) and eight microphysical processes related to graupel at $48 \mathrm{~h}$. It can be seen that four processes are dominant: $G_{S}(S C), G_{c}, V_{G}(s u b)$, and $\mathrm{R}_{\mathrm{G}}$ (melt). Under the assumption that neither autoconversion from snow nor accretion of snow by graupel occurs, the initial formation is primarily caused by $\mathrm{G}_{\mathrm{S}}(\mathrm{SC})$, accretion of cloud water by snow. Once some amount of graupel is 


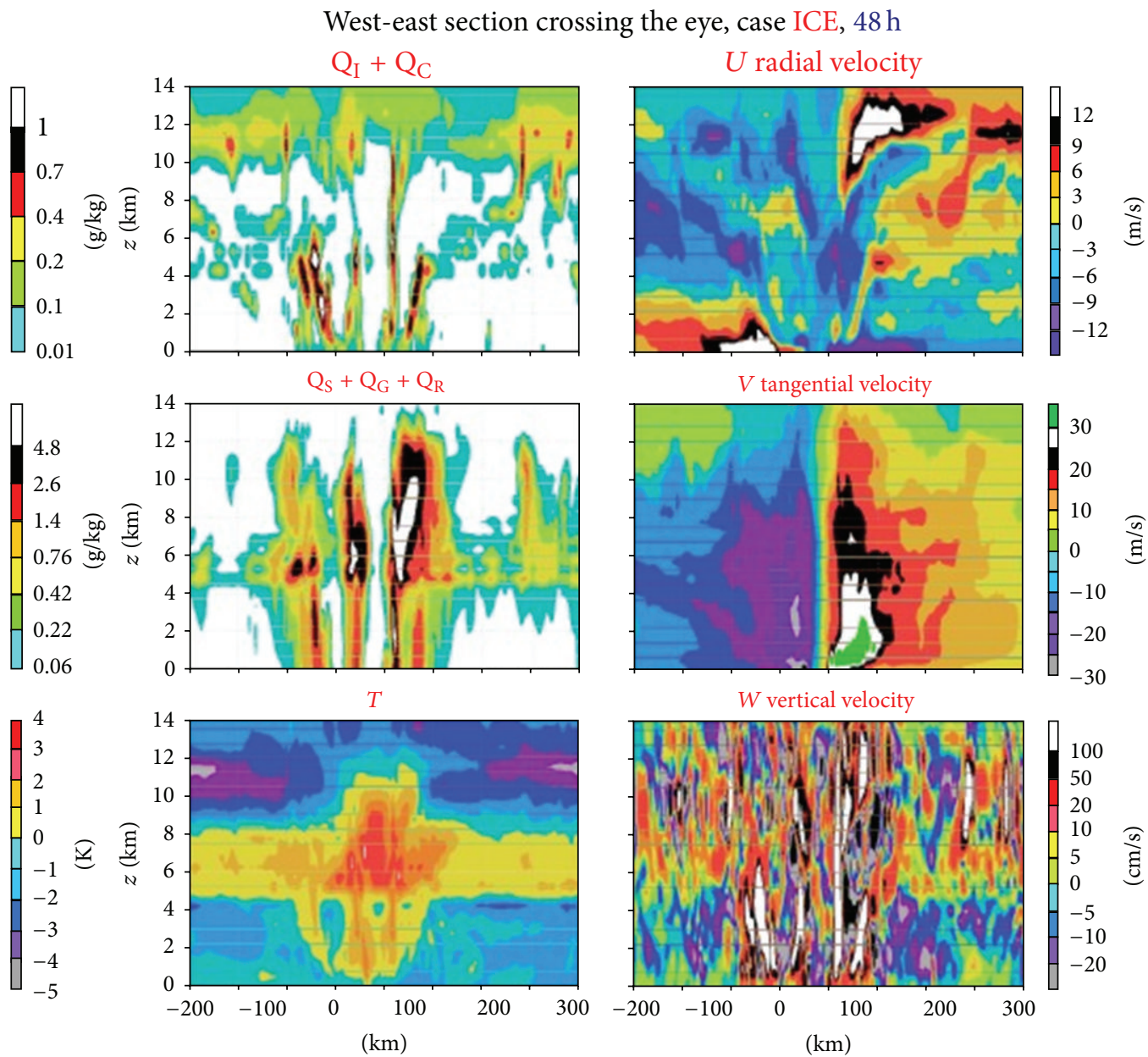

FIGURE 8: Vertical cross section (west-east section) of nonprecipitating substance, that of precipitating substance, temperature anomaly, radial velocity, tangential velocity, and vertical velocity at $48 \mathrm{~h}$ in case ICE. The section crosses the center of the eye which is shown in the middle panel of Figure 7.

formed, $G_{c}$ (accretion of cloud water by graupel) becomes the most important process for graupel increase. Graupel is converted to rainwater by melting $\left(\mathrm{R}_{\mathrm{G}}\right.$ (melt)) and to water vapor by sublimation $\left(\mathrm{V}_{\mathrm{G}}(\mathrm{sub})\right)$. Deposition of water vapor by graupel $\left(\mathrm{G}_{\mathrm{V}}(\mathrm{dep})\right)$, accretion of cloud ice by graupel $\left(\mathrm{G}_{\mathrm{I}}\right)$, and accretion of rainwater by snow $\left(G_{S}(R S)\right.$ and $\left.G_{R}(R S)\right)$ are small.

The upper-left panel of Figure 11 shows the vertical crosssection of the mixing ratio of cloud ice at $48 \mathrm{~h}$. The middle-left panel shows $\mathrm{I}_{\mathrm{V}}$ (con), cloud ice formation from water vapor, which indicates condensation or sublimation. The vertical cross section of this quantity is somewhat similar to that of cloud ice mixing ratio, as expected. As for the warm rain process, the results are similar to what are known well. The mixing ratio of cloud water is shown in the upper-middle panel, and net condensation $\left(\mathrm{C}_{\mathrm{V}}(\mathrm{con})\right)$ is below this panel. The mixing ratio of rainwater is shown in the upper-right panel. Rainwater is produced by autoconversion from cloud water $\left(\mathrm{R}_{\mathrm{C}}\right.$ (auto)). Once some amount of rainwater is produced, accretion of cloud water by rainwater $\left(\mathrm{R}_{\mathrm{C}}\right)$ becomes the dominant process of rainwater increase. The effect of snow $\left(R_{C}(S)\right)$ is very small compared with $R_{C}$. Rainwater is converted to water vapor by evaporation $\left(\mathrm{V}_{\mathrm{R}}(\mathrm{evp})\right)$, which is the most important process to produce a cold pool in the subcloud layer.

The relative magnitude of cloud microphysical processes suggests which processes we should treat carefully or which parameterization formula we should examine for improvements. On the other hand, unimportant processes can be excluded in the numerical model to save computational time. Numerical experiments are also necessary to understand the role of each process and the model sensitivity to it. For instance, two numerical experiments have been performed with Kessler's and Berry's parameterizations for autoconversion from cloud water to rainwater. In the results presented in this paper, Kessler's parameterization (autoconversion threshold of $0.5 \mathrm{~g} \mathrm{~kg}^{-1}$ ) is used. An essentially similar result is obtained for Berry's parameterization. Other numerical experiments indicate that model results are not sensitive to the threshold value of autoconversion from cloud ice to snow and to the coefficient of the melting rate of the snow and graupel. On the other hand, the density of snow is important, 

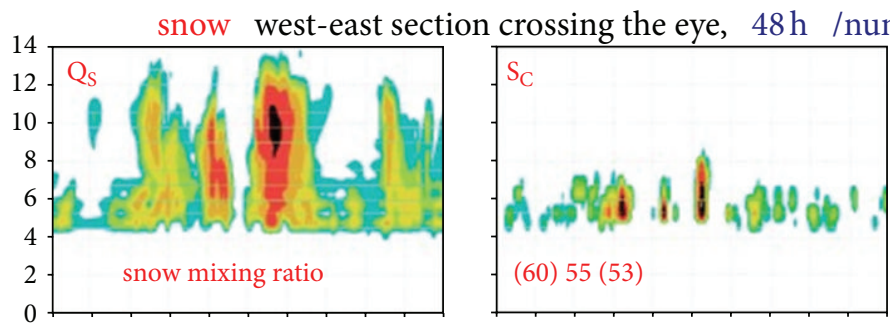

(60) $55(53)$
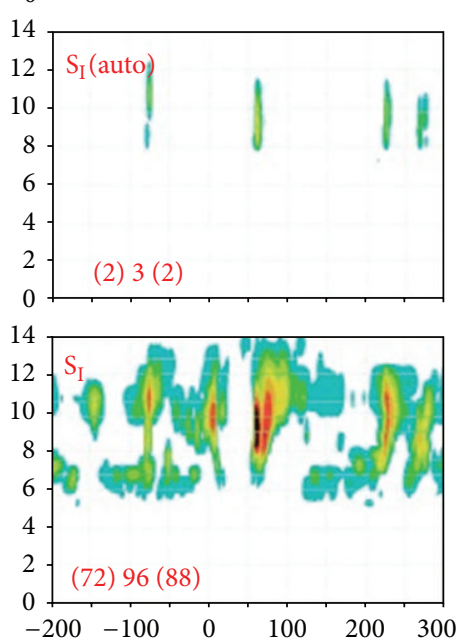
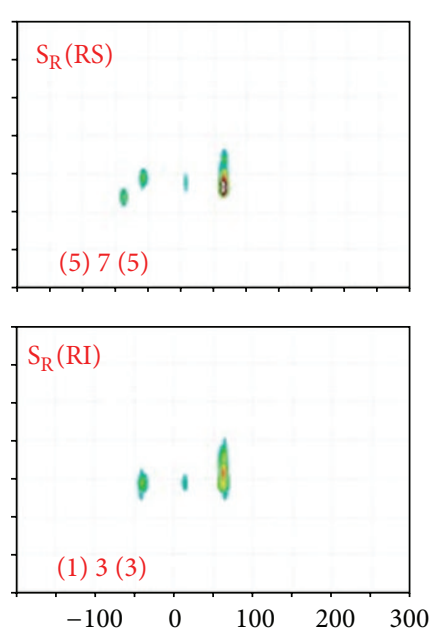

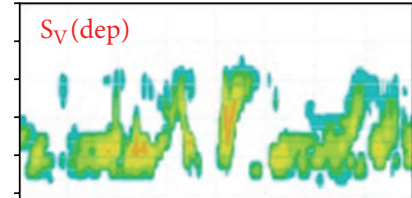

(86) $62(69)$
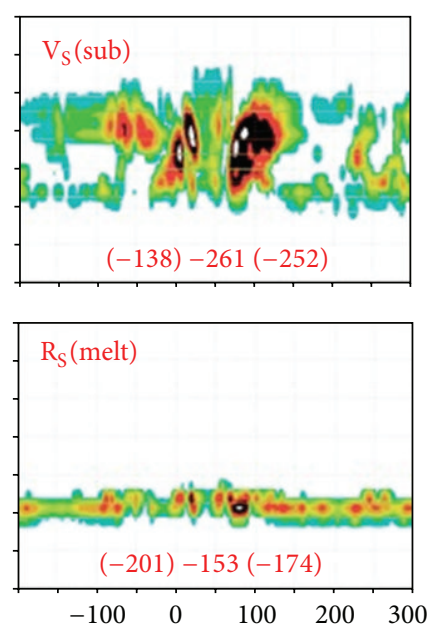

FIGURE 9: Vertical cross section (west-east section) of the mixing ratio of snow (upper-left panel) and eight processes of the cloud microphysics related to snow (other panels) at $48 \mathrm{~h}$ in case ICE. The section crosses the center of the eye. Three numerals in each panel indicate the magnitude of each process averaged in the area enclosed by the red square in Figure 6, at $36 \mathrm{~h}, 48 \mathrm{~h}$, and $58 \mathrm{~h}$. See text for $\mathrm{S}_{\mathrm{I}}\left(\right.$ auto), $\mathrm{S}_{\mathrm{I}}$, and others.

graupel west-east section crossing the eye, $48 \mathrm{~h} \quad$ /numerals ( $36 \mathrm{~h}) 48 \mathrm{~h}(58 \mathrm{~h})$
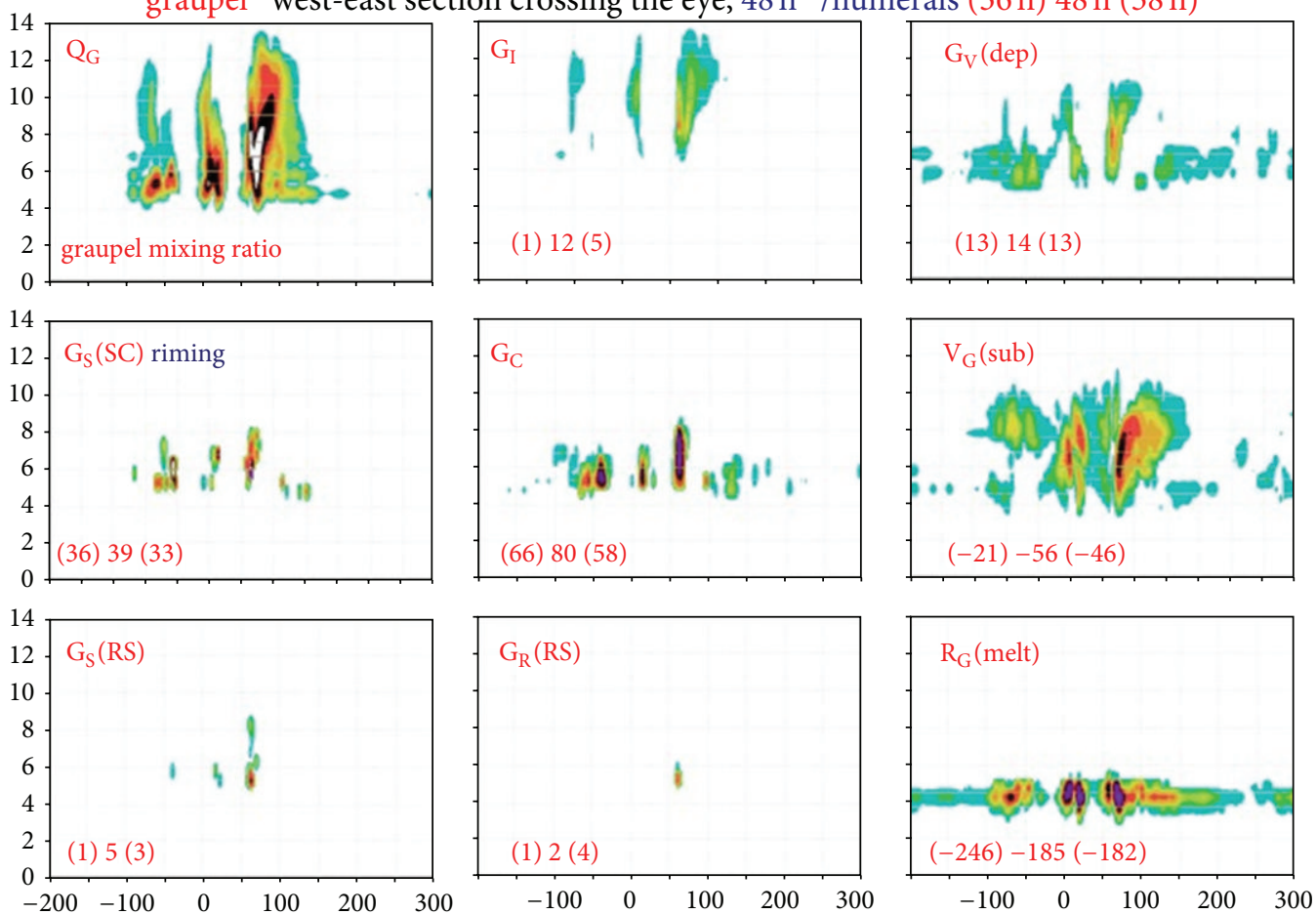

FIGURE 10: Same as Figure 9 but for graupel and cloud microphysics related to graupel. 


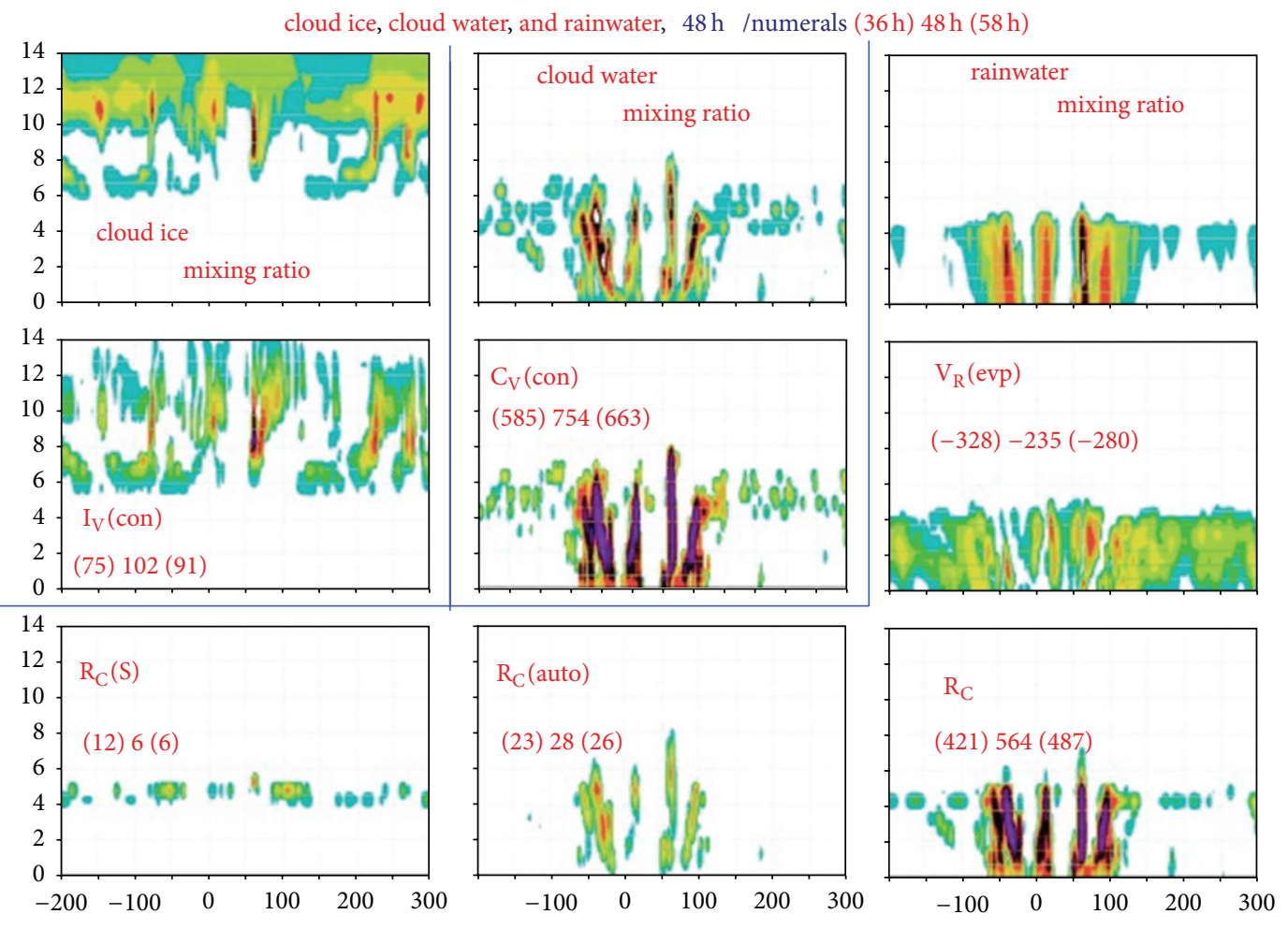

FIGURE 11: Same as Figure 9 but for cloud ice, cloud water, and rainwater.

particularly to the amount of cloud ice. This amount is 2 times larger in the case of the snow density of $0.3 \mathrm{~g} \mathrm{~cm}^{-3}$ than $0.1 \mathrm{~g} \mathrm{~cm}^{-3}$ because of the difference between the fall velocities. Further extensive studies on various problems remain to be made in the future.

\section{Concluding Remarks}

In order to understand why vortex (tropical cyclone) development is slower in the presence of the ice phase (Lord et al. [14]; Sawada and Iwasaki [15]), numerical experiments have been performed with and without the ice phase (case ICE and case NICE). It is shown that much more production of precipitating water substance in the mid-troposphere is most important to the slower development. In case ICE, falling snow and graupel melt, and rainwater evaporates in (and above) the subcloud layer. Evaporative cooling in case ICE is much larger than that in case NICE. More evaporative cooling produces stronger downdraft and cold pool, which makes the outward propagation of convection faster and the low-level pressure gradient smaller. The central surface pressure fall and the maximum wind intensification become slower in case ICE. As for the amounts of low-level rainwater (or surface rainfall intensity), these do not differ much among the two cases NICE and ICE.

Under the initial condition such that an axisymmetric vortex is given, as in the previous studies, convection takes a ring shape. The ring-shaped convection propagates outwards for the initial wind speed of $10 \mathrm{~ms}^{-1}$. The ring shape is maintained for a long period of time in case ICE, whereas in case NICE, it is destroyed in a short time, and the low-level air with high equivalent potential temperature enters easily the central area of the vortex through areas between spiralshaped convection. The central surface pressure falls and the maximum wind speed increases more rapidly in case NICE. The earlier realization of asymmetry in case NICE is partly due to the latitudinal variation of the Coriolis parameter, which was not taken into account in the previous studies.

Since the behavior of convection depends on the initial condition, further studies with respect to this point have also been made. The result will be reported in a separate paper, although the slower development of the vortex (TC) in the presence of the ice phase can be explained in a way similar to that described in this paper.

The effect of the melting of snow and graupel is also examined in this study. It is shown that this effect is small on the intensities of the downdraft and cold pool and the development of the vortex (TC), although it has a significant effect on the temperature field in the melting layer. It is also important to note that the melting has a large effect on the low-level precipitation distribution (pattern).

In order to improve parameterization scheme of the ice microphysical processes in the future, the relative importance of the processes is also examined and described in this paper. This problem remains to be studied.

\section{Acknowledgments}

The numerical experiments have been performed with the use of the NEC SX-8R supercomputer in the Japan Agency 
for Marine-Earth Science and Technology. The author wishes to thank one of the anonymous reviewers for his comments to improve the paper.

\section{References}

[1] K. Ooyama, "A dynamical model for the study of tropical cyclone development," Geofísica Internacional, vol. 4, pp. 187198, 1964.

[2] K. Ooyama, "Numerical simulation of the life cycle of tropical cyclones," Journal of the Atmospheric Sciences, vol. 26, pp. 3-40, 1969.

[3] J. G. Charney and A. Eliassen, "On the growth of the hurricane depression," Journal of the Atmospheric Sciences, vol. 21, pp. 6875, 1964.

[4] M. Yamasaki, "Numerical simulation of tropical cyclone development with the use of primitive equations," Journal of the Meteorological Society of Japan, vol. 46, pp. 178-201, 1968.

[5] M. Yamasaki, "A tropical cyclone model with parameterized vertical partition of released latent heat," Journal of the Meteorological Society of Japan, vol. 46, pp. 202-214, 1968.

[6] S. L. Rosenthal, "A circularly symmetric primitive equation model of tropical cyclone development containing an explicit water vapor cycle," Monthly Weather Review, vol. 98, pp. 643663, 1970.

[7] M. Yamasaki, "A further study of the tropical cyclone without Parameterizing the effects of cumulus convection," Papers in Meteorology and Geophysics, vol. 34, pp. 221-260, 1983.

[8] M. Yamasaki, "Parameterization of cumulus convection in a tropical cyclone model, collection of papers presented at the WMO/IUGG NWP Symposium, Tokyo, 1986," Journal of the Meteorological Society of Japan, vol. 65, pp. 665-678, 1987.

[9] M. Yamasaki, "A view on tropical cyclones as CISK," Journal of the Meteorological Society of Japan, vol. 85, pp. 145-164, 2007.

[10] M. Yamasaki, "A preliminary experiment of the tropical cyclone without parameterizing the effects of cumulus convection," Journal of the Meteorological Society of Japan, vol. 55, pp. 11-31, 1977.

[11] M. Yamasaki, "The role of surface friction in tropical cyclones," Journal of the Meteorological Society of Japan, vol. 55, pp. 559$572,1977$.

[12] H. E. Willoughby, H.-L. Jin, S. J. Lord, and J. M. Piotrowicz, "Hurricane structure and evolution as simulated by an axisymmetric, nonhydrostatic numerical model," Journal of the Atmospheric Sciences, vol. 41, no. 7, pp. 1169-1186, 1984.

[13] M. Yamasaki, Typhoon, Tokyodo Publishers, Tokyo, Japan, 1982.

[14] S. J. Lord, H. E. Willoughby, and J. M. Piotrowicz, "Role of a parameterized ice-phase microphysics in an axisymmetric, nonhydrostatic tropical cyclone model," Journal of the Atmospheric Sciences, vol. 41, no. 19, pp. 2836-2848, 1984.

[15] M. Sawada and T. Iwasaki, "Impacts of ice phase processes on tropical cyclone development," Journal of the Meteorological Society of Japan, vol. 85, no. 4, pp. 479-494, 2007.

[16] T. Zhu and D.-L. Zhang, "Numerical simulation of Hurricane Bonnie (1998). Part II: sensitivity to varying cloud microphysical processes," Journal of the Atmospheric Sciences, vol. 63, no. 1, pp. 109-126, 2006.

[17] M. Yamasaki, "A study of the 22 February 1993 TOGA COARE squall line: a case used for the GCSS model intercomparison," Journal of the Meteorological Society of Japan, vol. 82, no. 5, pp. 1331-1363, 2004.
[18] D. Sato and M. Yamasaki, "Effects of a squall cluster on the large-scale field and cloud microphysical processes" (Japanese), Presented at a spring meeting of the Meteorological Society of Japan, P266, 1998.

[19] D. Sato and M. Yamasaki, "A formulation of cloud ice formation and representation of graupel in a model of tropical squall clusters" (Japanese), Presented at a fall meeting of the Meteorological Society of Japan, B152, 1998.

[20] D. Sato and M. Yamasaki, "An improvement of parameterization of cloud microphysics-application to convection in tropical cyclones" (Japanese), Presented at a fall meeting of the Meteorological Society of Japan, B107, 1999.

[21] Y.-L. Lin, R. D. Farley, and H. D. Orville, "Bulk parameterization of the snow field in a cloud model," Journal of Climate \& Applied Meteorology, vol. 22, no. 6, pp. 1065-1092, 1983.

[22] M. Murakami, "Numerical modeling of dynamical and microphysical evolution of an isolated convective cloud-the 19 July 1981 CCOPE cloud," Journal of the Meteorological Society of Japan, vol. 68, pp. 107-128, 1990. 

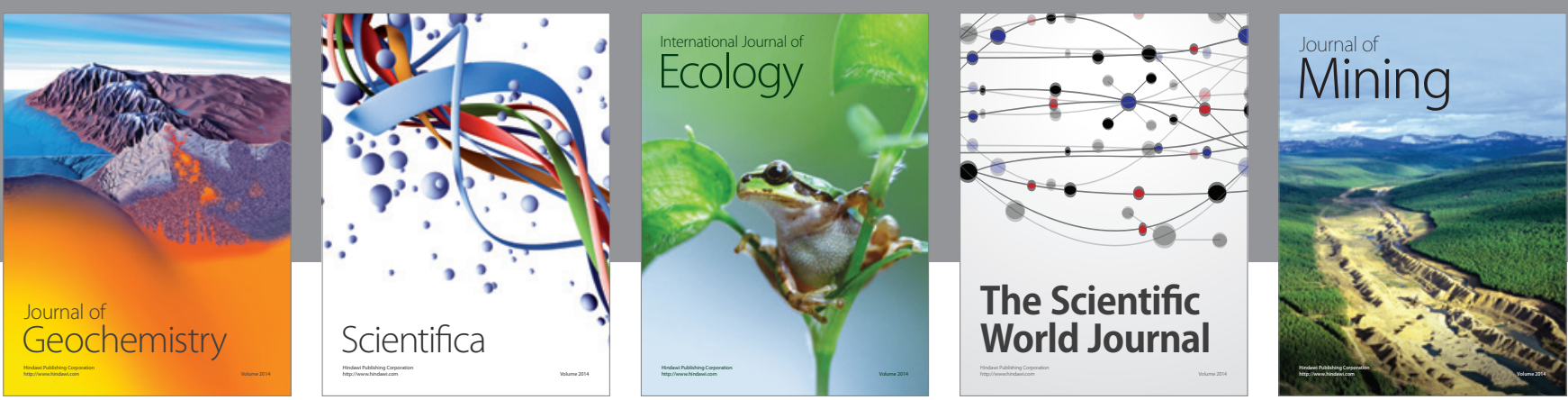

The Scientific World Journal
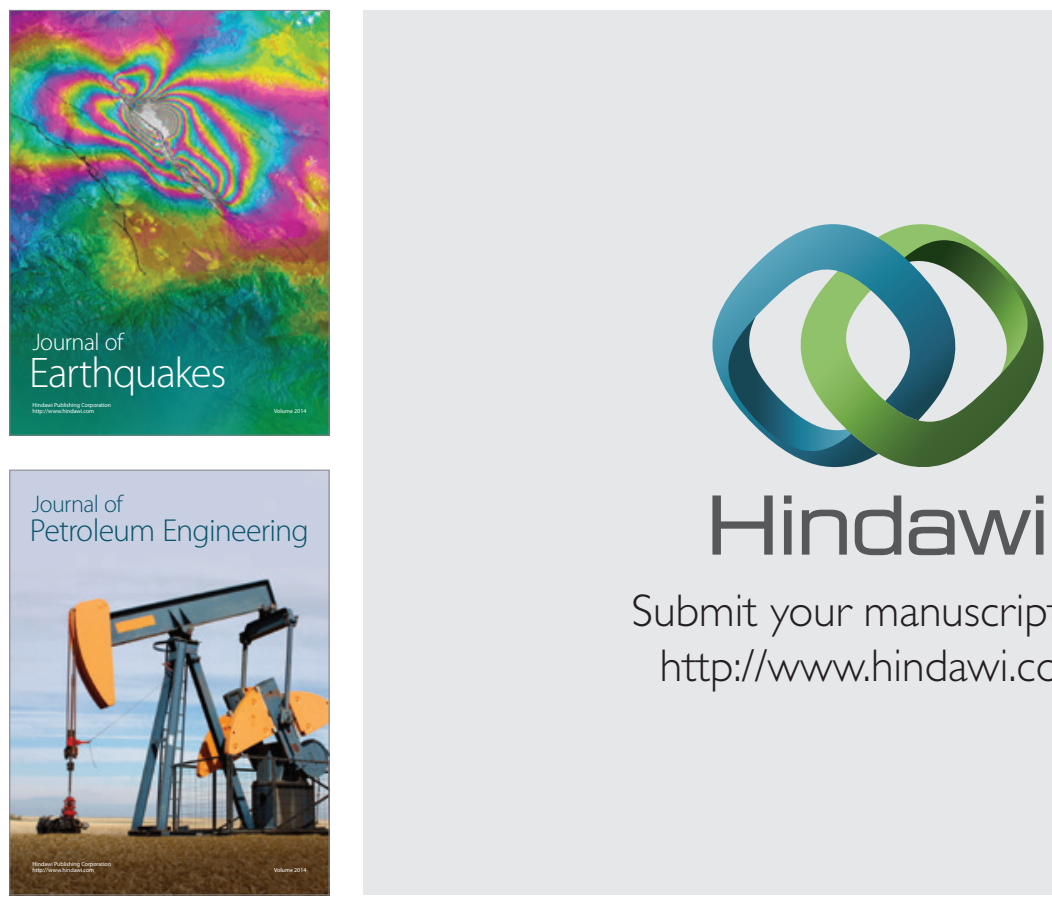

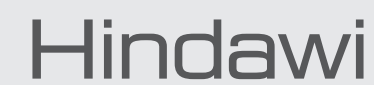

Submit your manuscripts at

http://www.hindawi.com
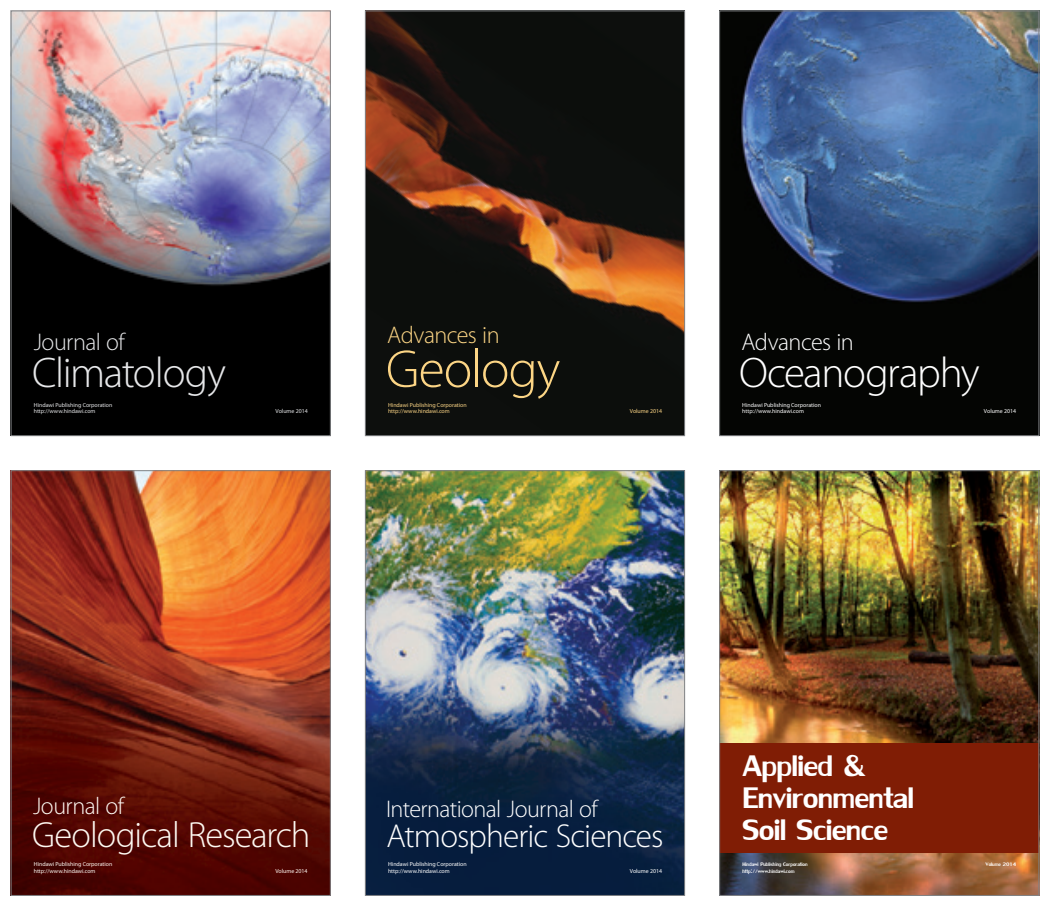
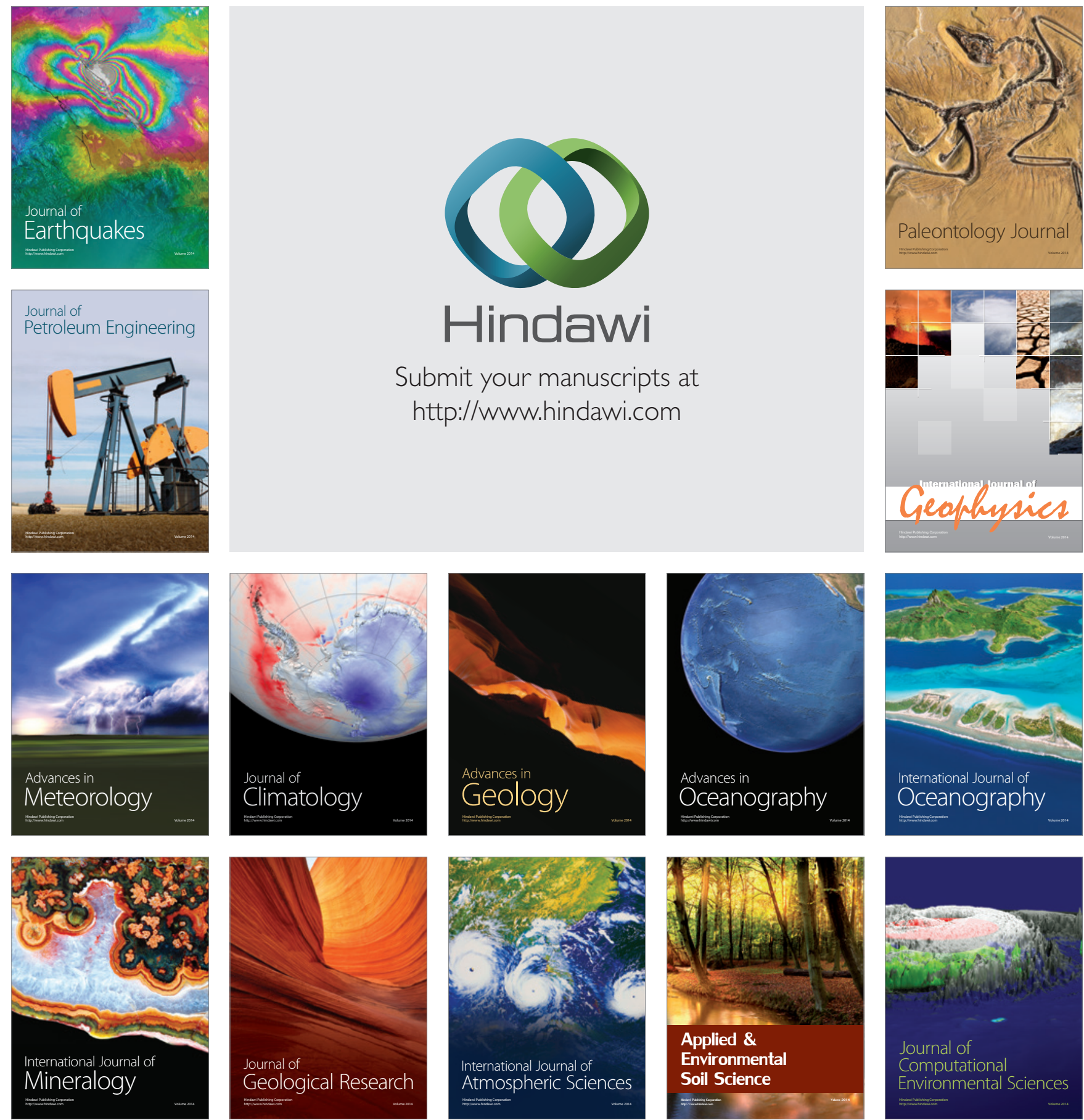